\title{
Dose-optimization in nuclear cardiac imaging, time for the next step?
}

\author{
Joris D. van Dijk, MSc, PhD \\ a Department of Nuclear Medicine, Isala Hospital, Zwolle, The Netherlands
}

Received Sep 4, 2018; accepted Sep 4, 2018

doi:10.1007/s12350-018-1441-2

\section{See related article, pp. 1974-1980}

Equilibrium radionuclide angiography (ERNA), also known as multigated acquisition (MUGA), is a well-validated non-invasive test to accurately determine the cardiac function. It is recommended in patients receiving potentially cardiotoxic chemotherapies and it is also used for the evaluation of prognosis in patients with cardiac diseases. ${ }^{1-3}$ ERNA is performed by labeling either erythrocytes or human serum albumin (HSA) with technetium-99m-pertechnetate (Tc-99m). After administration of the radioactive tracer, an electrocardiograph (ECG)-triggered planar projection is made of the chest using a gamma camera to determine the volume and contours of both ventricles. Next, a region of interest is drawn over the ventricles in the acquired phase images to calculate the ventricular function parameters using dedicated software.

ERNA was first introduced in the 1970s and rapidly became the standard technique for left ventricular ejection fraction (LVEF) assessment due to its high accuracy. ${ }^{4}$ Despite the emerging routine use of gated myocardial perfusion imaging (MPI) SPECT, magnetic resonance imaging and the increased availability of ultrasound, the relatively low operator dependence, low costs, and high reproducibility still make ERNA very valuable in common day practice to assess global heart performance. ${ }^{3,5}$

Although ERNA is widely used to derive the LVEF, it is also associated with a relatively high radiation dose of 3.5-7.4 mSv and protocols for LVEF determination have remained fairly unchanged since the introduction

Reprint requests: Joris D. van Dijk, MSc, PhD, Department of Nuclear Medicine, Isala Hospital, Zwolle, The Netherlands; jorisvdijk@gmail.com

J Nucl Cardiol 2019;26:1981-3.

$1071-3581 / \$ 34.00$

Copyright (C) 2018 American Society of Nuclear Cardiology. in the 1970s. ${ }^{4}$ This is remarkable, as radiation awareness has increased considerably over the last decades and the performance of the gamma cameras has improved substantially. ${ }^{6,7}$ Whereas early studies used $925 \mathrm{MBq}(25$ $\mathrm{mCi}$ ) Tc-99m, the dose reference levels are still around $800 \mathrm{MBq}(22 \mathrm{mCi})$ for an ERNA scan and the international guidelines published in 2006 and 2008 still recommend activities of $500-1050 \mathrm{MBq}$ (13$30 \mathrm{mCi}) .{ }^{1,2,4,8}$

An alternative to planar-ERNA is ERNA using ECG-gated SPECT. ERNA-SPECT has the possibility to calculate the ejection fraction of both left and right ventricle, to assess the ventricular motion and also to assess wall thickening. Although LVEF measurements using planar and SPECT imaging show a high correlation, ERNA-SPECT results in 7-10 \%-point higher values. ${ }^{9,10}$ As the evaluation of cardiotoxicity of chemotherapy was originally validated using planarERNA, one should therefore be cautious in interchanging the SPECT- and planar-ERNA measurements.

ERNA-SPECT is often performed with conventional Tc-99m activities whereas during the last decades improvements in soft- and hardware resulted in either an increase in image quality or an activity reduction in other nuclear cardiac SPECT studies. ${ }^{7,11}$ In terms of software, the routine use of iterative reconstruction and resolution recovery methods and the implementation of noise reduction algorithms enabled activity reductions without compromising image quality. In terms of hardware, especially the dedicated cardiac cadmium zinc telluride (CZT) based SPECT cameras have resulted in major improvements due their inherently high sensitivity and spatial resolution compared to conventional SPECT scanners with sodium-iodide scintillation crystals. ${ }^{11,12}$ Duvall et al. reported that reliable LVEF measurements can be obtained using $40 \%$ less activity with a CZTbased SPECT camera than with planar-ERNA. ${ }^{13}$ Moreover, they also reported that activity reductions of $50 \%$ are feasible when increasing the scan time from five to ten minutes. Despite these efforts, there is still a lot to 
gain by transforming fixed protocols into patient-specific activity protocols when comparing it to myocardial perfusion imaging (MPI) with SPECT. ${ }^{11}$

In the current issue of the Journal of Nuclear Cardiology, Rydberg et al. studied the influence of patient characteristics such as weight, height, and age on the count rate in ERNA using a CZT-based SPECT camera to derive a patient-specific activity protocol for better radiation dose justification. ${ }^{14}$ They retrospectively included 1065 patients who all underwent LVEF determination using ERNA-CZT-SPECT- using $550 \mathrm{MBq}$ Tc-99m-labeled human serum albumin. In their regression analysis they found that body weight explained $68 \%$ of the measured count rate. They further showed that their model could be improved to explain $75 \%$ of the count rate variation by also including height (4.5\%), gender $(3.2 \%)$, and age $(0.7 \%)$. Although they did not test their derived protocol in clinical practice, they did derive a patient-specific activity formula incorporating all these variables as a function of the desired count rate.

Although Rydberg et al. made a significant step in improving the acquisition protocol for SPECT-ERNA, the authors used count rate and not the cumulative number of measured counts as the acquisition time solely depended on the number of accepted beats. Although they showed that heart rate did not significantly influence the count rate, it is likely that the heart rate influences the total amount of counts accumulated as a lower heart rate will increase the scan time and, hence, the cumulative number of counts. Yet a higher number of cumulative counts will result, just like for MPI SPECT, in an improved image quality. Duvall et al. showed that an increasing scan time and, hence, increasing count statistics, resulted in a better correlation between planarERNA and ERNA-CZT-SPECT LVEF measurements. ${ }^{13}$ This seems in line with the current study by Rydberg et al. who showed that also the reproducibility of LVEF measurements was better in patients with higher count rates. It is therefore safe to say that a minimum number of cumulative counts is required to obtain reliable LVEF measurements. Acquiring this minimal number of counts can easily be achieved in all patients by administering a patient-specific activity.

Despite the count rate limitation, Rydberg et al. made an important step towards deriving an optimal patient-specific protocol for ERNA-SPECT and their methodology can also easily be used to derive an optimized activity protocol for planar-SPECT. Yet two additional steps have to be taken prior to clinical adoption, as previously described in a 'hands on approach' by van Dijk et al. ${ }^{15}$ The first step is to validate if the derived protocol also results in a constant image quality where the cumulative number of counts could be taken as a surrogate. The obtained constant number of counts also allows to reduce the average administered activity as current fixed-activity protocols are often aimed to achieve sufficient image quality in all, but especially in heavier patients. Leaner patients often receive an activity which is unnecessarily high using fixed-activity protocols and patient-specific protocols allow to reduce this activity. Hence, the second step is to determine the minimal number of cumulative counts required to obtain accurate and reliable LVEF measurements in all patients. This minimal number of required counts will vary between SPECT cameras and is influenced by reconstruction software and settings. Centers should therefore always test the effects of possible reductions for each new camera set-up before implementing it in clinical practice to ensure sufficient quality.

Besides activity protocol optimization, several other considerations should be made to deliver optimal patient care. First of all, selection of patients undergoing certain diagnostic testing should always be in concordance with the appropriate use criteria. ${ }^{16}$ Second, when the newest hard- and software that can cope with lower counting statistics are available, it should be used to further reduce the activity to administer. ${ }^{7}$ Third, the acquisition should be performed according to the guidelines using the correct patient positioning and camera angles. Final, as reproducibility of the post-processing of the data varies between centers, especially for ERNA-SPECT, ${ }^{17}$ one should be certain that standardized protocols are used to derive reliable and reproducible LVEFs.

In summary, in addition to the existing patientspecific activity protocols commonly used for MPI SPECT, Rydberg et al. demonstrated that patientspecific protocols will presumably also result in constant quality and better radiation dose justification in ERNA using CZT-SPECT. It therefore seems likely that tailored protocols may also result in better quality and activity optimization in other nuclear cardiac examinations such as planar-ERNA. It may therefore not only be time to adopt patient-specific activity protocols in SPECT-MPI and ERNA-SPECT, but also to derive, validate, and adopt patient-tailored protocols in other nuclear procedures.

\section{Disclosure}

Joris D. van Dijk has nothing to disclose.

\section{References}

1. Corbett JR, Akinboboye OO, Bacharach SL, et al. Equilibrium radionuclide angiocardiography. J Nucl Cardiol 2006;13:164. 
2. Hesse B, Lindhardt TB, Acampa W, et al. EANM/ESC guidelines for radionuclide imaging of cardiac function. Eur J Nucl Med Mol Imaging 2008;35:851-85.

3. Russell RR, Alexander J, Jain D, et al. The role and clinical effectiveness of multimodality imaging in the management of cardiac complications of cancer and cancer therapy. J Nucl Cardiol 2016;23:856-84.

4. Folland ED, Hamilton GW, Larson SM, et al. The radionuclide ejection fraction: a comparison of three radionuclide techniques with contrast angiography. J Nucl Med 1977;18:1159-66.

5. Mitra D. Equilibrium radionuclide angiocardiography: Its usefulness in current practice and potential future applications. World J Radiol 2012;4:421.

6. Gupta A, Bajaj NS. Reducing radiation exposure from nuclear myocardial perfusion imaging: Time to act is now. J Nucl Cardiol 2017;24:1856-9.

7. DePuey EG. Advances in SPECT camera software and hardware: currently available and new on the horizon. J Nucl Cardiol 2012;19:551-81

8. Administration of Radioactive Substances Advisory Committee (ARSAC). Notes for guidance on the clinical administration of radiopharmaceuticals and use of sealed radioactive sources. PHE publications gateway number 2017760: 2018.

9. Groch MW, DePuey EG, Belzberg AC, et al. Planar imaging versus gated blood-pool SPECT for the assessment of ventricular performance: a multicenter study. J Nucl Med 2001;42:1773-9.

10. Bartlett ML, Srinivasan G, Barker WC, et al. Left ventricular ejection fraction: comparison of results from planar and SPECT gated blood-pool studies. J Nucl Med 1996;37:1795-9.
11. van Dijk JD, Borren NM, Mouden M, et al. Effect of a patientspecific minimum activity in stress myocardial perfusion imaging using CZT-SPECT: Prognostic value, radiation dose, and scan outcome. J Nucl Cardiol 2018;25:26-35.

12. Esteves FP, Raggi P, Folks RD, et al. Novel solid-state-detector dedicated cardiac camera for fast myocardial perfusion imaging: Multicenter comparison with standard dual detector cameras. J Nucl Cardiol 2009;16:927-34.

13. Duvall WL, Guma-Demers KA, George T, Henzlova MJ. Radiation reduction and faster acquisition times with SPECT gated blood pool scans using a high-efficiency cardiac SPECT camera. J Nucl Cardiol 2016;23:1128-38.

14. Rydberg J, Andersen J, Haarmark C, Zerahn B. The influence of anthropometric and basic circulatory variables on count rate in cadmium-zinc-telluride SPECT gated radionuclide angiography. J Nucl Cardiol 2018

15. Van Dijk JD, Jager PL, Ottervanger JP, et al. Patient-specific tracer activity in MPI SPECT: A hands-on approach. J Nucl Cardiol 2016;23:145-8

16. Hendel RC, Berman DS, Di Carli MF, et al. ACCF/ASNC/ACR/ AHA/ASE/SCCT/SCMR/SNM 2009 appropriate use criteria for cardiac radionuclide imaging. J Am Coll Cardiol 2009;53:220129.

17. Trägårdh E, Ljungberg M, Edenbrandt L, et al. Evaluation of interdepartmental variability of ejection fraction and cardiac volumes in myocardial perfusion scintigraphy using simulated data. EJNMMI Phys 2015;2:2. 